\title{
Editorial
}

\section{Echocardiographic evaluation of ventricular diastolic function: implications for treatment}

Symptoms of left ventricular failure may occur in patients with normal or near normal systolic performance. ${ }^{12}$ Recent ACC/AHA guidelines for the evaluation and management of heart failure suggest that abnormal systolic and diastolic function should be separately assessed, because treatments differ. ${ }^{3}$ What, therefore, should the clinician attempt to measure when evaluating patients with ventricular disease? Can diastolic heart disease of different types be identified and can specific treatment be chosen on the basis of such measurements? Experience of systolic disease, where drugs aimed at increasing ventricular contractility were found to have little consistent effect on exercise tolerance and to have an adverse effect on outcome, suggests that answers to these questions cannot be taken for granted. ${ }^{4-6}$

\section{Diastolic function}

Diastole is usually taken to include the periods of isovolumic relaxation and ventricular filling: both may be abnor$\mathrm{mal}$ in patients with ventricular disease. Diastolic function differs from systole in being more sensitive to age, in being profoundly dependent on loading conditions, and in frequently reflecting the consequences of disturbances originating earlier in the cardiac cycle. Isovolumic relaxation time, taken as the interval between aortic valve closure and the onset of mitral cusp separation, is an excellent example of these interrelations. It is shortened by an increase in left atrial pressure and lengthened by ventricular disease..$^{7-9}$ Thus isovolumic relaxation time at any particular time represents a balance between underlying ventricular disease and loading conditions. In an individual patient it may vary from abnormally short to abnormally long without any basic change in cardiac state.

\section{Diastolic disease}

The underlying process of myocardial relaxation is usually assessed from the rate of decline of ventricular pressure. It can be estimated by reconstructing equivalent pressures from the continuous wave Doppler curve of mitral regurgitation, from which the time constant of pressure fall can be derived. ${ }^{1011}$ However, in ventricular disease, relaxation itself is ill-defined. In some patients, notably a few of those with left ventricular hypertrophy, the rate of tension decline may perhaps be uniformly reduced throughout the myocardium. Much the commonest cause of a reduced rate of pressure fall, though, is incoordination due to regional differences in the time of onset and the rate of tension decline. Incoordination cannot be distinguished from uniformly slow relaxation by any quality of the ventricular pressure, but only by detecting regional abnormal wall motion during isovolumic relaxation. Activation disturbances cause incoordinate relaxation independent of intrinsic myocardial disease. The extent to which pharmacological manipulation of abnormal relaxation is translated into patient benefit is still uncertain. Verapamil may shorten a prolonged isovolumic relaxation time in hypertrophic cardiomyopathy, but this is probably the result of an increase in left atrial pressure caused by its negative inotropic effect. Beneficial effects of diltiazem in dilated cardiomyopathy are likely to reflect a direct effect on afterload. In coronary artery disease, though, incoordinate relaxation regularly improves with successful angioplasty. ${ }^{12}$

\section{Ventricular filling}

Ventricular filling is totally determined by loading conditions. By Newton's second law of motion, atrioventricular force or pressure gradient, expressed in $\mathrm{mm} \mathrm{Hg} / \mathrm{cm}$, is the only factor that causes blood to move. Ventricular disease modifies filling only insofar as it modifies the atrioventricular pressure gradient. It is thus unfortunate that Doppler measurements of the ventricular filling pattern are so widely believed to reflect ventricular "diastolic function". Relations between loading conditions and flow velocities are complex. Blood acceleration not blood velocity is proportional to the atrioventricular pressure gradient. Peak blood velocity thus depends not only on pressure gradient, but also on the time over which it has acted. Pressure gradients vary during rapid filling, not only rapidly with time, as atrial and ventricular pressures approach one another, but also in space. Colour Doppler $M$ mode shows that the time of onset of atrioventricular flow, and thus of atrioventricular pressure reversal, is delayed by about $50 \mathrm{~ms}$ per $\mathrm{cm}$ in healthy people, and more in patients with ventricular disease.

\section{Assessing the effects of treatment}

The interpretation of measurements of diastolic function is thus far more complex than many clinicians would like, and their relation to treatment indirect at best. Experience from treating systolic disease suggests that the direct aim of treatment should not be to correct abnormal measurements of function. Rather, we suggest an alternative approach: that of identifying the changes in diastolic measurements occurring with treatment that are known to be effective in heart failure-that is, to increase exercise tolerance or improve prognosis.

Reducing raised left atrial pressure consistently increases exercise tolerance. When left atrial pressure is high, isovolumic relaxation time is abnormally short, Doppler E wave amplitude is increased, with a third heart sound occurring at its peak and its deceleration time reduced. The systolic component of pulmonary venous flow is reduced. As left atrial pressure falls, all these changes are reversed. Isovolumic relaxation time increases, becoming first normal and then abnormally prolonged. Cavity shape may change during isovolumic relaxation, often in a very striking manner, because of incoordination. On transmitral Doppler, E wave amplitude falls (often becoming suppressed altogether), the 
third sound and functional mitral regurgitation regress, and the $\mathrm{A}$ wave becomes much more prominent. Thus successful treatment with an ACE inhibitor may cause measurements of diastolic function to become more abnormal, as the patient's clinical state and prognosis improve. Failure of these changes to occur may suggest inadequate treatment. Conversely, when left atrial pressure is normal, isovolumic relaxation time is likely to be prolonged and E/A reduced. Such patients respond poorly to vasodilators. ${ }^{13}$ When a raised left atrial pressure is detected (therefore, particularly in patients with normal systolic function) and when the complex changes associated with its fall are being documented, echocardiographic measurements may be useful in determining the nature and effectiveness of treatment.

The major component of stroke volume may enter the ventricle with atrial systole in patients with ventricular disease, particularly when filling pressure is normal. Echocardiography allows such patients to be identified and treatment modified as necessary. It also presents a number of non-invasive approaches to studying atrial function. The timing of atrial systole is apparent on transmitral Doppler. The pattern of atrial contraction can be studied at multiple sites on the atrioventricular rings. Assessing atrial function is also very helpful, not only in deciding whether to institute DDD or simple VVI pacing, but also to set an appropriate value for atrioventricular delay to allow for the variable interatrial conduction time associated with pacing from the right atrial appendix.

In dilated cardiomyopathy, functional mitral regurgitation may be greatly prolonged, sometimes to $600 \mathrm{~ms}$ or more, by disturbances of activation. ${ }^{14}{ }^{15}$ Rates of ventricular pressure fall are reduced, so that filling time becomes very short. In such patients, restoring a more normal pattern of left ventricular activation by right ventricular pacing substantially shortens the mitral regurgitation, increases stroke volume, and sometimes doubles filling time. Objectively measured exercise capacity, both short and long term, is improved. ${ }^{16}$ Echo-Doppler is essential to identify and monitor such patients, who constitute $10-15 \%$ of those presenting with severe limitation of exercise tolerance caused by dilated cardiomyopathy.

Abnormal late diastolic function is usually caused by increased cavity stiffiness, itself the result of abnormal cavity architecture or abnormal myocardial fibrosis. This pathological process is not susceptible to direct treatment, though it can be palliated to some extent by reducing cavity size with a vasodilator. Restrictive cardiomyopathy must therefore be distinguished from constrictive pericarditis, which can be treated by surgery. Several approaches have been described. In pericardial constriction, the ventricular filling pattern changes strikingly with respiration, whereas with restrictive filling it is effectively fixed. In the superior vena cava, the $X$ descent is accentuated with constriction, and the $\mathrm{Y}$ descent with restriction. In both cavae, systolic flow towards the heart is maintained with constriction and effectively lost with restriction. In a few cases with valvar regurgitation, superimposed restrictive ventricular disease leads to symptoms developing when the retrograde flow, though not severe in volume terms, causes high filling pressures. This may lead to a decision for earlier surgery than otherwise would have been the case.

\section{Conclusions}

Diastolic disturbances should always be sought in the symptomatic patient in whom systolic function is normal, though treatment directed simply towards correcting them is unlikely to be satisfactory in the long term. One of the most effective methods of improving measurements of diastolic function is to raise left atrial pressure. The main contribution of pharmacology in diastolic disease is to reduce a raised filling pressure by manipulating loading conditions. If it is not raised, vasodilators will cause side effects. Echocardiography is sensitive in detecting a raised atrial pressure and in documenting the complex events that occur in abnormal ventricles as it falls, allowing the effects of treatment to be monitored. Pacing has a smaller role in treatment, but can manipulate the timing of left atrial systole and partially correct very abnormal activation in some patients with dilated cardiomyopathy. As more pieces of the jigsaw fit together, it may be possible to tailor therapy more specifically to diastolic abnormalities. At present, however, this desirable end has yet to be achieved.

Cardiac Department, St George's Hospital,

STEPHEN J D BRECKER Blackshaw Road, London SW17 OQT

Cardiac Department, The Royal Brompton Hospital,

DEREK G GIBSON Sydney Street, London SW3 $6 N P$

1 Soufer R, Wohlgelernter D, Vita NA, Amuchestegui M, Sostman HD, Berger HJ, et al. Intact systolic left ventricular function in clinical congestive heart failure. Am 7 Cardiol 1985;55:1032-6.

2 Marantz PR, Tobin JN, Wassertheil-Smoller S, Steingart RM, Wexler JP, Budner N, et al. The relationship between left ventricular systolic function and congestive heart failure diagnosed by clinical criteria. Circulation 1988;77:607-12

3 Williams JF, Jr, Bristow MR, Fowler MB, Francis GS, Garson A, Jr, Gersh $\mathrm{BJ}$, et al. Guidelines for the evaluation and management of heart failure. Report of the American College of Cardiology/American Heart Association Task Force on Practice Guidelines (Committee on EvaluaAssociation Task Force on Practice Guidelines (Committee on Evalua1376-98.

$4 \mathrm{Katz}$ AM. Potential deleterious effects of inotropic agents in the therapy of chronic heart failure. Circulation 1986;73(suppl III):184-190.

5 Massie B, Bourassa M, DiBianco R, Hess M, Konstam M, Likoff M, Packer M. Long-term oral administration of amrinone for congestive heart failure: lack of efficacy in a multicenter controlled trial. Circulation 1985;71:963-71

6 Packer M, Carver JR, Rodeheffer RJ, Ivanhoe RJ, Di Bianco R, Zeldis SM, et al, for the PROMISE Study Research Group: Effect of oral milrinone on mortality in severe chronic heart failure. $N$ Engl $¥$ Med 1991;325:1468-75

7 Mattheos M, Shapiro E, Oldershaw PJ, Sacchetti R, Gibson DG. Noninvasive assessment of changes in left ventricular relaxation by combined invasive assessment of changes in left ventricular relaxation by combined

$8 \mathrm{Ng} \mathrm{KSK}$, Gibson DG. Relation of filling pattern to diastolic function in severe left ventricular disease. Br Heart $\mathcal{f}$ 1990;63:209-14.

9 Brecker SJD, Lee CH, Gibson DG. Relation of left ventricular isovolumic relaxation time and incoordination to transmitral Doppler filling patterns. Br Heart $\mathcal{F}$ 1992;68:567-73.

10 Chen C, Rodriguez L, Guerrero L, Marshall S, Levine RA, Weyman AE, et al. Noninvasive estimation of the instantaneous first derivative of left ventricular pressure using continuous-wave Doppler echocardiography. tricular pressure using contin
Circulation 1991;83:2101-10.

11 Chen C, Rodriguez L, Levine RA, Weyman AE, Thomas JD. Noninvasive measurement of the time constant of left ventricular relaxation using the continuous-wave Doppler velocity profile of mitral regurgitation. continuous-wave Doppler
Circulation 1992;86:272-8.

12 Henein MY, Priestly K, Davarashvili T, Buller N, Gibson DG. Early changes in left ventricular subendocardial function after successful coronary angioplasty. Br Heart $\mathcal{f}$ 1993;69:501-6.

13 Topol EJ, Traill TA, Fortuin NJ. Hypertensive hypertrophic cardiomyopathy of the elderly. N Engl ₹ Med 1985;312:277-83.

14 Brecker SJD, Xiao HB, Sparrow J, Gibson DG. Effects of dual-chamber pacing with short atrioventricular delay in dilated cardiomyopathy. Lancet 1992;340:1308-12.

15 Xiao HB, Brecker SJD, Gibson DG. Differing effects of right ventricular pacing and left bundle branch block on left ventricular function. $\mathrm{Br}$ Heart F 1993;69:166-73.

16 Brecker SJ, Kelly PA, Chua TP, Gibson DG. Effects of permanent dual chamber pacing in end-stage dilated cardiomyopathy (abstract). Circulation 1995;92(suppl I):I-724. 